\title{
von Kármán self-preservation hypothesis for magnetohydrodynamic turbulence and its consequences for universality
}

\author{
Minping Wan ${ }^{1}$, Sean Oughton ${ }^{2}$, Sergio Servidio ${ }^{3}$ and William H. Matthaeus ${ }^{1} \dagger$ \\ ${ }^{1}$ Bartol Research Institute and Department of Physics and Astronomy, University of Delaware, \\ Newark, DE 19716, USA \\ 2 Department of Mathematics, University of Waikato, Hamilton 3240, New Zealand \\ ${ }^{3}$ Dipartimento di Fisica, Universita' della Calabria, I-87036 Cosenza, Italy
}

(Received 11 May 2011; revised 31 October 2011; accepted 27 January 2012; first published online 6 March 2012)

We argue that the hypothesis of preservation of shape of dimensionless second- and third-order correlations during decay of incompressible homogeneous magnetohydrodynamic (MHD) turbulence requires, in general, at least two independent similarity length scales. These are associated with the two Elsässer energies. The existence of similarity solutions for the decay of turbulence with varying cross-helicity implies that these length scales cannot remain in proportion, opening the possibility for a wide variety of decay behaviour, in contrast to the simpler classic hydrodynamics case. Although the evolution equations for the second-order correlations lack explicit dependence on either the mean magnetic field or the magnetic helicity, there is inherent implicit dependence on these (and other) quantities through the third-order correlations. The self-similar inertial range, a subclass of the general similarity case, inherits this complexity so that a single universal energy spectral law cannot be anticipated, even though the same pair of third-order laws holds for arbitrary crosshelicity and magnetic helicity. The straightforward notion of universality associated with Kolmogorov theory in hydrodynamics therefore requires careful generalization and reformulation in MHD.

Key words: homogeneous turbulence, MHD turbulence, turbulence theory

\section{Introduction}

The von Kármán-Howarth equations for time evolution of the two-point, singletime correlation functions in homogeneous hydrodynamic turbulence (de Kármán \& Howarth 1938) can be employed as a starting point for obtaining at least several essential parts of classical turbulence theory. Notable among these are the famed Kolmogorov (1941a) ' $2 / 3$ law' for the second-order structure functions (equivalent to the '-5/3 law' for spectra, Obukhov 1941), the Kolmogorov (1941c) ' $4 / 5$ law' for the third-order structure functions, and von Kármán and Howarth's own similarity decay theory for the turbulence energy and similarity scale. Here we are concerned with the implications of the von Kármán-Howarth similarity hypothesis when applied 
to incompressible homogeneous three-dimensional (3D) magnetohydrodynamic (MHD) turbulence. Hereafter MHD will refer to the incompressible homogeneous 3D case, unless stated otherwise.

The extension of the von Kármán-Howarth approach to incompressible isotropic MHD was performed by Chandrasekhar $(1951 a, b)$, using the velocity and magnetic field variables. Much later, Politano \& Pouquet (1998a,b) derived the appropriate equations in terms of Elsässer variables, and also determined the associated third-order ('4/5') law for MHD. Energy decay laws based on simple (dimensional) analysis have been offered for MHD (Biskamp 1994; Hossain et al. 1995) and subjected to some numerical testing (Hossain et al. 1995; Politano, Pouquet \& Sulem 1995). Additional analysis along these lines has suggested dependence of energy decay on the lowwavenumber form of the spectrum (Galtier, Politano \& Pouquet 1997) and associated conservation laws for linear or angular momentum and self-similarity of the large scales (Davidson 2009, 2010). Other studies have extended the von Kármán-Howarth equations to helical MHD (Politano, Gomez \& Pouquet 2003) and to Hall MHD (Galtier 2008). There have also been numerous recent studies (MacBride, Forman \& Smith 2005; Podesta, Forman \& Smith 2007; Sorriso-Valvo et al. 2007; Podesta 2008; Carbone, Sorriso-Valvo \& Marino 2009; Wan et al. 2009) that examine the third-order MHD cascade law with non-zero cross-helicity. However, as far as we are aware the methodology of von Kármán and Howarth has not been employed to examine the consistency of the similarity structure of MHD energy decay (although see Davidson $(2009,2010)$ for related similarity work). In the present paper we carry out this analysis to examine the requirements of the self-preservation hypothesis as a route to obtaining a similarity solution for energy decay in MHD. We find here that such considerations introduce additional parameters in the similarity theories. This leads naturally to an enumeration of several quantities that may control the varieties of MHD turbulence and discussion of the possibly central role of the fourth-order correlations in determining the type of MHD turbulence that emerges in the dynamics.

For MHD systems with a mean magnetic field $\boldsymbol{B}_{0} \equiv B_{0} \hat{z}$, the turbulent fields become anisotropic (Robinson \& Rusbridge 1971; Shebalin, Matthaeus \& Montgomery 1983; Bondeson 1985; Carbone \& Veltri 1990; Oughton, Priest \& Matthaeus 1994). The nature of the anisotropy is such that the formation of gradients parallel to $\boldsymbol{B}_{0}$ is suppressed relative to those that form due to the cascade in the perpendicular directions. This anisotropy gives rise to special representations such as reduced MHD (Montgomery 1982; Zank \& Matthaeus 1992; Goldreich \& Sridhar 1995), in which turbulent spectral transfer is mainly in the perpendicular direction. Energy decay can also be influenced by anisotropy, since the dynamics becomes progressively more two-dimensional as $B_{0}$ increases. We address here the implications of the von Kármán-Howarth approach to the decay of energy in MHD with cross-helicity, for both the isotropic and anisotropic $\left(B_{0} \neq 0\right)$ cases, and several other issues that influence the prospects for the universality of the dynamical description in terms of similarity variables.

The paper is structured as follows. In $\S 2$ we review the derivation of the MHD von Kármán-Howarth equations. Sections 3 and 4 develop the conditions for similarity solutions when the flow has non-zero cross-helicity, the former for the isotropic case and the latter when there is a mean magnetic field. Section 5 briefly calls attention to the physical implications of the higher-order von Kármán-Howarth equations. Section 6 discusses the known varieties of MHD turbulence based on the global ideal invariants and other global parameters, contrasting with the 3D hydrodynamic case where it is often assumed that the only relevant global quantity is the energy (per 
unit mass). In $\S 7$ the prospects for a 'universal' description of MHD turbulence are discussed. We summarize the results in $\S 8$. In the Appendix, the evolution equations for the structure functions are given.

\section{2. von Kármán-Howarth equations for MHD}

We begin with a brief review of the development of the von Kármán-Howarth equations for homogeneous incompressible 3D MHD (Smith 1981; Politano \& Pouquet 1998a,b), which describe the evolution of the second-order correlation functions for that system. Incompressible MHD involves a velocity field $v_{i}$ and a magnetic field (in Alfvén speed units) $b_{i}$, for Cartesian components $i=1,2,3$. Both fields are solenoidal, e.g. $\nabla_{i} v_{i}=0$ (sum implied). We take the mass density $\rho=$ constant. For completeness we admit a uniform applied DC magnetic field $\boldsymbol{B}_{0}=B_{0} \hat{z}$ to include expected effects such as generation of anisotropy through suppression of the parallel cascade (Shebalin et al. 1983). In terms of the Elsässer variables $z^{ \pm}=\boldsymbol{v} \pm \boldsymbol{b}$, the MHD equations are

$$
\partial_{t} z_{i}^{ \pm}=-\left(z_{k}^{\mp} \mp B_{0 k}\right) \partial_{k} z_{i}^{ \pm}-\partial_{i} P+v \partial_{k} \partial_{k} z_{i}^{ \pm},
$$

where $P$ is the total pressure and $v$ is the kinematic viscosity, here assumed equal to the resistivity.

Let the unprimed variables $z^{ \pm}$denote the fields at position $x$ and the primed versions $z^{ \pm \prime}$ denote the fields at the displaced position $\boldsymbol{x}^{\prime}=\boldsymbol{x}+\boldsymbol{r}$, where $\boldsymbol{r}$ is the difference vector or spatial lag. Thus, at $\boldsymbol{x}^{\prime}$,

$$
\partial_{t} z_{i}^{ \pm \prime}=-\left(z_{k}^{\mp \prime} \mp B_{0 k}\right) \partial_{k}^{\prime} z_{i}^{ \pm \prime}-\partial_{i}^{\prime} P^{\prime}+v \partial_{k}^{\prime} \partial_{k}^{\prime} z_{i}^{ \pm \prime},
$$

where $\partial_{k}^{\prime}=\partial / \partial x_{k}^{\prime}$. It is straightforward to use (2.1) and (2.2) to compute the time derivative of the second-order correlation

$$
R_{i j}^{ \pm}(\boldsymbol{r}, t)=\left\langle z_{i}^{ \pm} z_{j}^{ \pm \prime}\right\rangle
$$

with the angular brackets denoting an ensemble average. One obtains the following von Kármán-Howarth equations for the evolution of the trace $R_{i i}^{ \pm}$

$$
\begin{aligned}
\partial_{t} R_{i i}^{ \pm} & =-\frac{\partial}{\partial r_{k}}\left[\left\langle z_{k}^{\mp \prime} z_{i}^{ \pm} z_{i}^{ \pm \prime}\right\rangle-\left\langle z_{k}^{\mp} z_{i}^{ \pm} z_{i}^{ \pm \prime}\right\rangle\right]+2 v \frac{\partial^{2} R_{i i}^{ \pm}}{\partial r_{k} \partial r_{k}} \\
& =-\frac{\partial}{\partial r_{k}}\left[\hat{Q}_{k}^{ \pm}(\boldsymbol{r})-\hat{Q}_{k}^{ \pm}(-\boldsymbol{r})\right]+2 v \frac{\partial^{2} R_{i i}^{ \pm}}{\partial r_{k} \partial r_{k}} .
\end{aligned}
$$

Here we have introduced the triple correlations

$$
\hat{Q}_{k}^{ \pm}(\boldsymbol{r}) \equiv\left\langle z_{k}^{\mp \prime} z_{i}^{ \pm} z_{i}^{ \pm \prime}\right\rangle,
$$

and made use of spatial homogeneity to deduce that $\left\langle z_{k}^{\mp} z_{i}^{ \pm} z_{i}^{ \pm \prime}\right\rangle=\hat{Q}_{k}^{ \pm}(-\boldsymbol{r})$. These triple correlations are time dependent, although this is not indicated in the notation here. One can demonstrate that the pressure terms do not contribute to (2.5) (Politano \& Pouquet $1998 b$ ), as is also the case for hydrodynamics (de Kármán \& Howarth 1938).

It is noteworthy that $\boldsymbol{B}_{0}$ does not appear in the second-order equation (2.5). This seems paradoxical since it is well documented that a DC magnetic field has an influence on the overall energy decay and also the spectral distribution of energy (e.g. Shebalin et al. 1983; Bondeson 1985; Grappin 1986; Carbone \& Veltri 1990; Oughton et al. 1994). As we discuss below, the resolution is that the third-order correlations $\hat{Q}_{k}^{ \pm}$have an implicit dependence on $\boldsymbol{B}_{0}$, as is apparent from their own 
evolution equations (see $\S 5$ ). Thus, through higher-order equations the lifetime of the triple correlations comes to depend upon $\boldsymbol{B}_{0}$, and this dependence influences the second-order correlations. As is well known, such dependence of the triple lifetimes on $\boldsymbol{B}_{0}$ is a key factor in phenomenologies for the energy spectrum in MHD turbulence (e.g. Iroshnikov 1963; Kraichnan 1965; Montgomery 1982; Matthaeus \& Zhou 1989; Goldreich \& Sridhar 1995).

For completeness, we recall that the above von Kármán-Howarth equations can be restated in terms of structure functions such as $\left\langle\left|\delta z_{i}^{ \pm}\right|^{2}\right\rangle$, where $\delta z^{ \pm}=\boldsymbol{z}^{ \pm}\left(\boldsymbol{x}^{\prime}\right)-\boldsymbol{z}^{ \pm}(\boldsymbol{x})$ are the field increments. See the Appendix and Politano \& Pouquet $(1998 a, b)$ and Wan et al. (2009).

\section{Similarity decay in isotropic MHD with cross-helicity}

In this section, we derive the self-similar decay equations for isotropic MHD with non-zero cross-helicity, $H_{c}=\langle\boldsymbol{v} \cdot \boldsymbol{b}\rangle$. The equations for the zero cross-helicity situation can be recovered as a special case. Since we are considering isotropic systems, we set $\boldsymbol{B}_{0}=\boldsymbol{0}$. Here we define isotropic to mean that the statistical properties of the system are independent of rotations of the coordinate system, with no restriction on the system's mirror symmetry. Note that some older works (e.g. de Kármán \& Howarth 1938; Robertson 1940; Kolmogorov 1941a; Chandrasekhar 1951a,b) include mirror symmetry as part of their definition of isotropy.

We start from the von Kármán-Howarth equation (2.5). For isotropic MHD this can be further simplified, since when the statistical properties are independent of rotations we have $R_{i i}^{ \pm}(\boldsymbol{r}, t)=R_{i i}^{ \pm}(r, t)$, with $r=|\boldsymbol{r}|$. Similarly, isotropy also requires that

$$
\hat{Q}_{k}^{ \pm}(\boldsymbol{r})=Q^{ \pm}(r) \hat{r}_{k}
$$

where $\hat{\boldsymbol{r}}=\boldsymbol{r} /|\boldsymbol{r}|$ and the $Q^{ \pm}(r)$ are even functions of $r$ (Batchelor 1970). Then (2.5) becomes

$$
\partial_{t} R_{i i}^{ \pm}(r, t)=-2 \frac{\partial Q^{ \pm}(r)}{\partial r}-4 \frac{Q^{ \pm}}{r}+2 v\left[\frac{\partial^{2} R_{i i}^{ \pm}}{\partial r^{2}}+\frac{2}{r} \frac{\partial R_{i i}^{ \pm}}{\partial r}\right] .
$$

Following de Kármán \& Howarth (1938) we now adopt the hypothesis of selfpreservation of the correlation function during the decay of turbulence. A natural set of similarity variables are the associated Elsässer energies (per unit mass) $Z_{ \pm}^{2}=\left\langle z^{ \pm}(\boldsymbol{x}) \cdot z^{ \pm}(\boldsymbol{x})\right\rangle$ and the similarity length scales $L^{ \pm}$, usually associated with the energy-containing or outer scale for each Elsässer field.

Let us focus first on the correlation functions associated with the 'plus' Elsässer field. Provided that $L^{+} \gg \lambda_{\text {diss }}$ the (Kolmogorov) dissipation scale, and $L^{+}$also is well separated from the system size, one may posit that $L^{+}$is the only relevant length. In this case, the similarity form of the correlation is

$$
R_{i i}^{+}(r, t)=Z_{+}^{2}(t) f(\eta)
$$

where the dimensionless spatial lag is $\eta \equiv r / L^{+}(t)$. Note that $\eta$ is an implicit function of time. Using (3.3), we have

$$
\partial_{t} R_{i i}^{+}=\frac{\mathrm{d} Z_{+}^{2}}{\mathrm{~d} t} f-Z_{+}^{2} f^{\prime} \frac{\eta}{L^{+}} \frac{\mathrm{d} L^{+}}{\mathrm{d} t}
$$


where $f^{\prime} \equiv \mathrm{d} f / \mathrm{d} \eta$. We assume that the required third-order correlation can also be written in a similarity form

$$
Q^{+}(r)=Z_{-} Z_{+}^{2} q(\eta),
$$

from which it follows that

$$
\frac{\partial Q^{+}(r)}{\partial r}=Z_{-} Z_{+}^{2} \frac{\mathrm{d} q(\eta)}{\mathrm{d} r}=\frac{Z_{-} Z_{+}^{2}}{L^{+}} q^{\prime} .
$$

Here the form $q(\eta)$ is motivated by consideration of the structure of the nonlinear terms in the MHD equations: $\dot{z}_{+}^{2} \sim z_{-} \cdot \nabla z_{+}^{2}$. This suggests that any dependence of the triple correlation on $L^{-}$is implicit. Other assumptions are possible, e.g. one might presume that the 'minus' length scale would also appear explicitly so that $q=q\left(\eta, r / L^{-}\right)$. This form leads to $L^{+} \propto L^{-}$and $Z_{+} \propto Z_{-}$, a case discussed later in this section.

With the aid of (3.4) and (3.6), we can reassemble (3.2) as

$$
\begin{gathered}
\left\{\frac{\mathrm{d} Z_{+}^{2}}{\mathrm{~d} t}\right\}[f]-\left\{\frac{Z_{+}^{2}}{L^{+}} \frac{\mathrm{d} L^{+}}{\mathrm{d} t}\right\}\left[f^{\prime} \eta\right]+\left\{2 \frac{Z_{-} Z_{+}^{2}}{L^{+}}\right\}\left[q^{\prime}\right]+\left\{4 \frac{Z_{-} Z_{+}^{2}}{L^{+}}\right\}\left[\frac{q}{\eta}\right] \\
-\left\{2 v \frac{Z_{+}^{2}}{L^{+2}}\right\}\left[f^{\prime \prime}\right]-\left\{4 v \frac{Z_{+}^{2}}{L^{+2}}\right\}\left[\frac{f^{\prime}}{\eta}\right]=0 .
\end{gathered}
$$

Under the assumptions of the similarity variables that we adopted, the explicit time dependence of the correlations occurs only through the similarity variables $Z_{+}^{2}(t)$ and $L^{+}(t)$. The dimensionless correlation functions $f(\eta)$ and $q(\eta)$ depend only upon the dimensionless variable $\eta$ and thus lack explicit time dependence. Consequently, the terms in (3.7) that are enclosed in curly braces $\{\ldots\}$ are explicitly time dependent, while the terms enclosed in square brackets [...] are time independent (or, more precisely, only depend on time implicitly, through $\eta$ ). If a similarity solution to the equations exists, the solutions would contain, for example, the potentially universal form of the dimensionless correlation functions such as $f(\eta)$. We note now, again following von Kármán and Howarth, that such solutions will exist only in the case that the time-dependent coefficients in each term remain in constant proportion to one another. In that case the time dependence can be eliminated in favour of the constants of proportionality, and the sought after similarity solutions would then be obtained. For the above case, the required auxiliary conditions are

$$
\frac{\mathrm{d} Z_{+}^{2}}{\mathrm{~d} t} \propto \frac{Z_{+}^{2}}{L^{+}} \frac{\mathrm{d} L^{+}}{\mathrm{d} t} \propto \frac{Z_{-} Z_{+}^{2}}{L^{+}},
$$

from which we obtain

$$
\frac{\mathrm{d} L^{+}}{\mathrm{d} t}=c_{1} Z_{-}, \quad \frac{\mathrm{d} Z_{+}^{2}}{\mathrm{~d} t}=c_{2} \frac{Z_{-} Z_{+}^{2}}{L^{+}},
$$

where $c_{1}$ and $c_{2}$ are constants, with $c_{2}<0$ since the energy should decay. Note that (3.8) omits the term involving the viscosity $\nu$, so that we are considering self-similarity for the inviscid MHD von Kármán-Howarth equation. If we insist that the viscous terms are also included in the similarity decay analysis, then an additional relationship is found, namely, $\mathrm{d} L^{+} / \mathrm{d} t=c_{5} v / L_{+}$. Along with the first relation in (3.9) this implies that $L^{+} Z_{-}=c^{\prime} v$, with $c^{\prime}$ a constant. If we take $R^{+}=L^{+} Z_{-} / v$ as an estimate of the Reynolds number during decay of the $Z_{+}$amplitude, the above relation would imply constant Reynolds number during the decay. This is a more robust type of similarity 
decay, originally suggested by von Kármán \& Lin (1949). Also, see Speziale \& Bernard (1992) for a discussion of the so-called 'partial' similarity solutions versus the 'complete' similarity solutions in which the viscous term is retained.

The above procedure can be directly repeated for the decay of the minus Elsässer energy $Z_{-}^{2}(t)$, introducing also its associated similarity scale $L^{-}(t)$. The dimensionless correlation functions (not shown) are assumed to depend on a similarity variable $\eta^{-}=r / L^{-}$. The conditions for a similarity solution become, in this case,

$$
\frac{\mathrm{d} L^{-}}{\mathrm{d} t}=c_{3} Z_{+}, \quad \frac{\mathrm{d} Z_{-}^{2}}{\mathrm{~d} t}=c_{4} \frac{Z_{+} Z_{-}^{2}}{L^{-}} .
$$

The constants $c_{1}-c_{4}$ are at this point undetermined. However, there is one property they must posses on fundamental grounds. If the original MHD equations admit a solution in terms of the functions $\{\boldsymbol{v}(t), \boldsymbol{B}(t)\}$, then the functions $\{\boldsymbol{v}(t),-\boldsymbol{B}(t)\}$ represent another solution. However, under this transformation, the plus and minus Elsässer fields are interchanged, and so also are $Z_{+} \leftrightarrows Z_{-}$and $L^{+} \leftrightarrows L^{-}$. Based on this symmetry we must impose that $c_{1}=c_{3}$ and $c_{2}=c_{4}$ in the similarity decay equations.

The energy decay equations for isotropic MHD given in (3.9) and (3.10) are not unfamiliar. In fact, the same or comparable equations for MHD energy decay have been written previously (Dobrowolny, Mangeney \& Veltri 1980; Hossain et al. 1995) on the basis of dimensional analysis or via modelling, sometimes motivated by similar approaches in hydrodynamics (e.g. Kolmogorov 1941b; Dryden 1943). Frequently the equations have involved only a single length scale $L$ (e.g. Biskamp 1994; Galtier et al. 1997) or have included a length scale for each Elsässer field, $L^{+}$and $L^{-}$, but then specialized to the case where $L^{+}=L^{-}=L$, so that only one scale remains. Still other related approaches (e.g. Matthaeus et al. 1994) have developed alternative qualitative arguments for the evolution of $L^{+}$and $L^{-}$that have not been equivalent to the above equations. An interesting alternative approach to discussion of similarity in energy decay is to relate this issue to the behaviour of the very low wavenumber region of the spectrum (Galtier et al. 1997, 1999). Some of these prior examinations of similarity in energy decay have allowed for differences in behaviour of the two Elsässer length scales, but appear not to have required such differences.

However, as shown above, (3.9) and (3.10) are requirements for a similarity solution and for consistency with the von Kármán hypothesis of self-preservation of the dimensionless correlation functions defined by (3.3) and (3.5) and their 'minus' analogues. Moreover, we now demonstrate that these equations require the presence of two independent length scales whenever the normalized cross-helicity is time-varying.

Suppose that $L^{+}(0)=L^{-}(0)=L(0)$. Then from (3.9) and (3.10), and using the symmetry of the constants, we obtain $\mathrm{d}\left(L^{+}-L^{-}\right) / \mathrm{d} t=c_{1}\left(Z_{-}-Z_{+}\right)$. Maintaining in time the condition that $L^{+}=L^{-}=L$ therefore requires that $Z_{+}(t)=Z_{-}(t)$ for all time. Consequently, employing a single similarity scale for decay of both Elsässer energies is consistent with self-preservation when the cross-helicity $Z_{+}^{2}-Z_{-}^{2} \propto\langle\boldsymbol{v} \cdot \boldsymbol{b}\rangle$ remains identically zero.

This result may be broadened somewhat. Suppose one stipulates that, instead of being equal, the two Elsässer lengths remain in proportion to one another, so that $L^{-} / L^{+}=\alpha$ is constant. Then from (3.9) and (3.10) we find immediately that $Z_{-} / Z_{+}=1 / \alpha$ so that the normalized cross-helicity $\sigma_{c}=\left(Z_{+}^{2}-Z_{-}^{2}\right) /\left(Z_{+}^{2}+Z_{-}^{2}\right)$ must also remain constant. We note in passing that if the more general form of similarity alluded to above following equation (3.9) is enforced, involving also the dissipative terms, then the assumption that $L^{-} / L^{+}=\alpha$, along with the extended set of similarity equations, leads to the conclusion that $\alpha=1$. This is the case already discussed above. It follows 
that in the more general case of non-constant $\sigma_{c}$, self-similar decay will require (at least) two similarity scales.

We are concerned here with investigating the existence of similarity solutions. However, it is important to acknowledge that even when they exist their physical relevance may be limited (e.g. Barenblatt 1996). For example, in hydrodynamic flows similarity solutions can be asymptotic to the long time behaviour, but may never actually manifest in some decaying flows at finite Reynolds number (e.g. Tennekes \& Lumley 1972). Simulations indicate that it typically takes one or two nonlinear times before similarity decay sets in, suggesting that turbulence correlations need to become properly established to support such similarity solutions. Moreover, higherorder moments will likely require still longer periods of time before they can be described by similarity solutions.

We close this section by recalling two further points regarding the isotropic case. First, there is the issue of local anisotropy (e.g. Cho \& Vishniac 2000; Milano et al. 2001), where it is argued that despite the global isotropy, various subregions have a well-defined (large-scale) local mean field which induces anisotropy in the small scales of the subregion. If the local anisotropy is present, it is in a randomly oriented coordinate system and is properly considered to be a higher-order quantity (that is, a property of higher-order moments). Consideration of this effect would take us beyond the scope of the current topics.

The second point is that there is a close structural relationship of the above similarity decay laws with the well-known third-order law that applies specifically to the inertial range in steady state and at high mechanical and magnetic Reynolds numbers. Politano \& Pouquet $(1998 a, b)$ extended Kolmogorov's third-order law for hydrodynamics (Kolmogorov 1941c; Frisch 1995) to MHD. Relationships of this type are written in terms of the spatial increments of the fluctuations $\delta z^{ \pm}=z^{ \pm}(x+r)-z^{ \pm}(x)$ (see also the Appendix). Without regard for rotational symmetry, the MHD equations under the above assumptions lead to

$$
\frac{\partial}{\partial r_{k}}\left\langle\delta z_{k}^{\mp}\left|\delta z_{i}^{ \pm}\right|^{2}\right\rangle=-4 \epsilon^{ \pm},
$$

where $\epsilon^{ \pm}$are the dissipation functions for the Elsässer fields. For the isotropic case that is relevant to the present section, the MHD third-order law further reduces to

$$
\left\langle\delta z_{L}^{\mp}\left|\delta z_{i}^{ \pm}\right|^{2}\right\rangle=-\frac{4}{3} \epsilon^{ \pm} r,
$$

where $\delta z_{L}^{ \pm}=\hat{\boldsymbol{r}} \cdot \delta z^{ \pm}$are the longitudinal increments.

To summarize, a similarity solution for decay of the Elsässer energies need not exist. However, the above development shows that for the isotropic case, existence of a similarity solution (with these assumed forms for the dimensionless functions) with non-constant $\sigma_{c}$ requires two similarity length scales, and not only one as typically employed in the hydrodynamic case.

\section{Self-similar decay with a mean magnetic field}

The focus of the present section is the prospect of a similarity description of energy decay in MHD turbulence with a mean magnetic field $\boldsymbol{B}_{0}$ (again allowing $H_{c} \neq 0$ ). In the introduction we briefly summarized some of the evidence that $\boldsymbol{B}_{0}$ induces a strong spectral anisotropy. It is quite reasonable to suppose that this would influence the energy decay rate. Simulations support this view (e.g. Oughton et al. 1994; Hossain et al. 1995) and suggest that non-zero $\boldsymbol{B}_{0}$ reduces energy decay relative to the isotropic 
case, but that the effect saturates at strong $\boldsymbol{B}_{0}$ (Hossain et al. 1995). On the other hand, it is not difficult to show that a uniform $\boldsymbol{B}_{0}$ does not enter into the (second-order) von Kármán-Howarth equations for MHD, as is evident from the derivation of (2.5). Furthermore, the differential form of the third-order law assumes the same form, (3.11), whether a mean magnetic field is present or not, as was shown by Politano \& Pouquet (1998a); see also Podesta et al. (2007).

It is worthwhile then to explore how the presence of anisotropy has an impact on realizing a similarity decay of the energy in MHD with a $\boldsymbol{B}_{0}$. We proceed by deriving the conditions for self-similar decay in anisotropic MHD with a mean magnetic field, starting from the von Kármán-Howarth equation (2.5). We again emphasize that (2.5) holds for any value of $B_{0}$, including zero.

Adopting the apparently inconsequential additional assumption of axisymmetry, we can write

$$
R_{i i}^{ \pm}(\boldsymbol{r}, t)=R_{i i}^{ \pm}\left(r_{\|}, r_{\perp}, t\right)
$$

where $r_{\|} \equiv \boldsymbol{r} \cdot \hat{z}$ and $r_{\perp} \equiv\left|\boldsymbol{r}-r_{\|} \hat{z}\right|$. These are standard cylindrical coordinates for the separation vector, with the azimuthal angle ignorable due to axisymmetry. Similarly, in these coordinates, and with axisymmetry, and invoking the theory of axisymmetric tensors (Batchelor 1970; Matthaeus \& Smith 1981) the vector mixed triple correlation defined in (2.6) can be written as

$$
\hat{Q}_{k}^{ \pm}(\boldsymbol{r})=A^{ \pm}\left(r_{\|}, r_{\perp}\right) \hat{r}_{k}+C^{ \pm}\left(r_{\|}, r_{\perp}\right) \hat{z}_{k},
$$

where the time dependence is not written. It immediately follows that (2.5) becomes

$$
\partial_{t} R_{i i}^{ \pm}=-\left(\frac{\partial A_{2}^{ \pm}}{\partial r_{\perp}} \frac{r_{\perp}}{r}+\frac{\partial A_{2}^{ \pm}}{\partial r_{\|}} \frac{r_{\|}}{r}+\frac{2 A_{2}^{ \pm}}{r}+\frac{\partial C_{2}^{ \pm}}{\partial r_{\|}}\right)+2 v\left(\frac{\partial^{2} R_{i i}^{ \pm}}{\partial r_{\perp}^{2}}+\frac{1}{r_{\perp}} \frac{\partial R_{i i}^{ \pm}}{\partial r_{\perp}}+\frac{\partial^{2} R_{i i}^{ \pm}}{\partial r_{\|}^{2}}\right)
$$

where

$$
\begin{aligned}
& A_{2}^{ \pm}\left(r_{\|}, r_{\perp}\right)=A^{ \pm}\left(r_{\|}, r_{\perp}\right)+A^{ \pm}\left(-r_{\|}, r_{\perp}\right), \\
& C_{2}^{ \pm}\left(r_{\|}, r_{\perp}\right)=C^{ \pm}\left(r_{\|}, r_{\perp}\right)-C^{ \pm}\left(-r_{\|}, r_{\perp}\right),
\end{aligned}
$$

The symmetries $A_{2}^{ \pm}\left(-r_{\|}, r_{\perp}\right)=A_{2}^{ \pm}\left(r_{\|}, r_{\perp}\right)$ and $C_{2}^{ \pm}\left(-r_{\|}, r_{\perp}\right)=-C_{2}^{ \pm}\left(r_{\|}, r_{\perp}\right)$, are evident.

In order to maintain the idea of self-preservation of the functional form of the two-point correlation during turbulent decay, we should be able to normalize this correlation to an energy and express the resulting dimensionless correlation as a function of a minimal number of scaled dimensionless coordinates. Perhaps the simplest assumption is that there are only two relevant similarity length scales for $Z^{+}$, say, $L_{\perp}^{+}$and $L_{\|}^{+}$associated with the directions perpendicular and parallel to $\boldsymbol{B}_{0}$. Under these conditions similarity decay of energy, if it occurs, would involve,

$$
R_{i i}^{+}(\boldsymbol{r}, t)=Z_{+}^{2}(t) f\left(\eta_{\|}, \eta_{\perp}\right),
$$

where $\eta_{\|} \equiv r_{\|} / L_{\|}^{+}(t)$ and $\eta_{\perp} \equiv r_{\perp} / L_{\perp}^{+}(t)$. Now we use (4.6) to write the left-hand side of (4.3) as

$$
\partial_{t} R_{i i}^{+}=\frac{\mathrm{d} Z_{+}^{2}}{\mathrm{~d} t}[f]-Z_{+}^{2}\left[\frac{\partial f}{\partial \eta_{\|}} \frac{\eta_{\|}}{L_{\|}^{+}} \frac{\mathrm{d} L_{\|}^{+}}{\mathrm{d} t}+\frac{\partial f}{\partial \eta_{\perp}} \frac{\eta_{\perp}}{L_{\perp}^{+}} \frac{\mathrm{d} L_{\perp}^{+}}{\mathrm{d} t}\right] .
$$

Employing also the similarity forms

$$
A_{2}^{+}\left(r_{\|}, r_{\perp}\right)=Z_{-} Z_{+}^{2} a\left(\eta_{\|}, \eta_{\perp}\right),
$$




$$
C_{2}^{+}\left(r_{\|}, r_{\perp}\right)=Z_{-} Z_{+}^{2} c\left(\eta_{\|}, \eta_{\perp}\right),
$$

we can reassemble (4.3) as

$$
\begin{aligned}
& \left\{\frac{\mathrm{d} Z_{+}^{2}}{\mathrm{~d} t}\right\}[f]-\left\{\frac{Z_{+}^{2}}{L_{\|}^{+}} \frac{\mathrm{d} L_{\|}^{+}}{\mathrm{d} t}\right\}\left[\frac{\partial f}{\partial \eta_{\|}} \eta_{\|}\right]-\left\{\frac{Z_{+}^{2}}{L_{\perp}^{+}} \frac{\mathrm{d} L_{\perp}^{+}}{\mathrm{d} t}\right\}\left[\frac{\partial f}{\partial \eta_{\perp}} \eta_{\perp}\right] \\
& +\left\{\frac{Z_{-} Z_{+}^{2}}{L_{\perp}^{+}}\right\}\left[\frac{1}{\sqrt{\eta_{\perp}^{2}+\alpha^{2} \eta_{\|}^{2}}}\left(\frac{\partial a}{\partial \eta_{\perp}} \eta_{\perp}+\frac{\partial a}{\partial \eta_{\|}} \eta_{\|}+2 a\right)\right]+\left\{\frac{Z_{-} Z_{+}^{2}}{L_{\|}^{+}}\right\}\left[\frac{\partial c}{\partial \eta_{\|}}\right] \\
& \quad-\left\{2 v \frac{Z_{+}^{2}}{L_{\|}^{+2}}\right\}\left[\frac{\partial^{2} f}{\partial \eta_{\|}^{2}}\right]-\left\{2 v \frac{Z_{+}^{2}}{L_{\perp}^{+2}}\right\}\left[\frac{\partial f}{\partial \eta_{\perp}} \frac{1}{\eta_{\perp}}+\frac{\partial^{2} f}{\partial \eta_{\perp}^{2}}\right]=0
\end{aligned}
$$

where now $\alpha=L_{\|}^{+} / L_{\perp}^{+}$. As in (3.5), here we also assume that the dimensionless 'plus' triple correlations, $a$ and $c$, depend explicitly only on the 'plus' length scales. Again, other assumptions are possible. Also note that in (4.10) we attempted to write curly braces $\{\cdots\}$ around all terms that vary in time, and square brackets $[\cdots]$ around terms that depend only on the dimensionless similarity variables $\eta_{\perp}$ and $\eta_{\|}$, in analogy to the procedure in $\S 3$ and in de Kármán \& Howarth (1938). However, one readily sees that this is not possible in general because the quantity $\alpha(t)$, the ratio of two similarity length scales, in general varies with time $t$. As such it seems that a similarity solution of this form exists only when $\alpha$ is a constant. This is the special case in which the length scales $L_{\perp}^{+}(t)$ and $L_{\|}^{+}(t)$, themselves time-varying, remain in constant proportion to one another throughout the period of self-similar turbulent decay. Thus, only one of these length scales should be considered as independent.

Supposing that the conditions exist for $\alpha$ to remain constant, we will have

$$
\frac{\mathrm{d} Z_{+}^{2}}{\mathrm{~d} t} \propto \frac{Z_{+}^{2}}{L_{\perp}^{+}} \frac{\mathrm{d} L_{\perp}^{+}}{\mathrm{d} t} \propto \frac{Z_{-} Z_{+}^{2}}{L_{\perp}^{+}},
$$

so that

$$
\frac{\mathrm{d} L_{\perp}^{+}}{\mathrm{d} t}=d_{1} Z_{-}, \quad \frac{\mathrm{d} Z_{+}^{2}}{\mathrm{~d} t}=d_{2} \frac{Z_{-} Z_{+}^{2}}{L_{\perp}^{+}},
$$

where $d_{1}$ and $d_{2}$ are constants. The analogous equations for the minus fields are easily obtained and, of course, are very similar to (4.12) and indeed (3.9)-(3.10). One then concludes that, just as in the isotropic case with $\sigma_{c}$ non-constant, similarity decay of the two Elsässer energies in anisotropic MHD with a mean magnetic field (and arbitrary $H_{c}$ ) involves two independent lengths. For the anisotropic case these may be taken to be $L_{\perp}^{+}$and $L_{\perp}^{-}$; the parallel length scales are fixed by $L_{\|}^{+}=\alpha L_{\perp}^{+}$, and $L_{\|}^{-}=\alpha L_{\perp}^{-}$, with $\alpha$ a constant.

The above condition of constant $\alpha$ seems very restrictive. One might suspect that the adopted representation in terms of similarity variables is too simple. It is possible to assume, instead of (4.6), a similarity representation that appears to be more general, such as

$$
R_{i i}^{+}(r, t)=Z_{+}^{2}(t) f\left(\eta_{\|}, \eta_{\perp}, \zeta\right),
$$

where $\zeta=Z_{+}^{2} /\left(Z_{+}^{2}+B_{0}^{2}\right)$. However, as we now demonstrate, this more general form changes only the time derivative of the second-order correlation, so that instead of the 
first three terms on the left-hand side of (4.10), upon using (4.13), we obtain

$$
\partial_{t} R_{i i}^{+}=\frac{\mathrm{d} Z_{+}^{2}}{\mathrm{~d} t}[f]-Z_{+}^{2}\left[\frac{\partial f}{\partial \eta_{\|}} \frac{\eta_{\|}}{L_{\|}^{+}} \frac{\mathrm{d} L_{\|}^{+}}{\mathrm{d} t}+\frac{\partial f}{\partial \eta_{\perp}} \frac{\eta_{\perp}}{L_{\perp}^{+}} \frac{\mathrm{d} L_{\perp}^{+}}{\mathrm{d} t}\right]+\frac{\mathrm{d} Z_{+}^{2}}{\mathrm{~d} t}\left[\zeta(1-\zeta) \frac{\partial f}{\partial \zeta}\right] .
$$

The first three terms are the same as above; only the fourth term, involving $\partial f / \partial \zeta$ is new. But this term multiplies $\mathrm{d} Z_{+}^{2} / \mathrm{d} t$ and therefore can be collected with the first term proportional to $f$. Consequently the conditions for maintaining a similarity solution are the same as those leading to (4.12), and the constant $\alpha$ condition relating $L_{\|}^{ \pm}$and $L_{\perp}^{ \pm}$ remains in place.

It is of course possible that other assumed forms for the dimensionless correlations might avoid this constant $\alpha$ restriction. The physical relevance of these postulated forms would also need to be verified. For the present, it is difficult to see how an anisotropic similarity decay solution in the presence of a mean magnetic field can be realized without enforcing $\alpha=$ constant.

Summarizing, a similarity solution for decay of the Elsässer energies when $B_{0} \neq 0$ need not exist. Such similarity solutions as we have been able to find require two independent similarity length scales, $L_{\perp}^{ \pm}$, with the parallel scales multiples of these. That is, we have found no solutions with a single similarity scale, nor any with independent perpendicular and parallel scales.

Finally in this section, we note that various spectral models of high crosshelicity (imbalanced) turbulence have been presented (Grappin, Pouquet \& Léorat 1983; Lithwick, Goldreich \& Sridhar 2007; Chandran 2008; Beresnyak \& Lazarian 2008, 2010; Perez \& Boldyrev 2009). It may be that the similarity results obtained herein are useful in further modelling of high- $H_{c}$ turbulence, particularly as regards the number of independent length scales.

\section{Role of the higher-order von Kármán-Howarth equations}

One of the goals of the present paper is to point out that several parameters need to be taken into account to differentiate various kinds of MHD turbulence. However the third-order law, whether in its integrated isotropic form (3.12) or the more general differential anisotropic form (3.11), does not contain these factors explicitly. There is clearly a strong implicit dependence, which influences spectral (second-order) statistics through the intermediary of the third-order mixed structure functions that appear in (3.11) and (3.12). This influence must enter explicitly at the higher orders. In particular, we can derive the evolution equations for third-order correlation functions $\hat{Q}_{k}^{ \pm}(\boldsymbol{r}, t)=\left\langle z_{k}^{\mp \prime} z_{i}^{ \pm} z_{i}^{ \pm \prime}\right\rangle$ (and their counterparts $\left\langle z_{k}^{\mp} z_{i}^{ \pm} z_{i}^{ \pm \prime}\right\rangle$ ):

$$
\begin{aligned}
\partial_{t}\left\langle z_{k}^{\mp \prime} z_{i}^{ \pm} z_{i}^{ \pm \prime}\right\rangle= & \frac{\partial}{\partial r_{m}}\left\langle z_{m}^{\mp} z_{k}^{\mp \prime} z_{i}^{ \pm} z_{i}^{ \pm \prime}\right\rangle-\left\langle z_{k}^{\mp \prime} \partial_{m}^{\prime}\left(z_{m}^{\mp \prime} z_{i}^{ \pm} z_{i}^{ \pm \prime}\right)\right\rangle-\left\langle z_{i}^{ \pm} z_{i}^{ \pm \prime} \partial_{m}^{\prime}\left(z_{m}^{ \pm \prime} z_{k}^{\mp \prime}\right)\right\rangle \\
& \mp 2 B_{0 m}\left\langle z_{i}^{ \pm} z_{i}^{ \pm \prime} \partial_{m}^{\prime} z_{k}^{\mp \prime}\right\rangle+\frac{\partial}{\partial r_{i}}\left\langle P z_{i}^{ \pm \prime} z_{k}^{\mp \prime}\right\rangle-\left\langle z_{k}^{\mp \prime} \partial_{i}^{\prime}\left(P^{\prime} z_{i}^{ \pm}\right)\right\rangle-\left\langle z_{i}^{ \pm} z_{i}^{ \pm \prime} \partial_{k}^{\prime} P^{\prime}\right\rangle \\
& +v \frac{\partial^{2}}{\partial r_{m}^{2}}\left\langle z_{k}^{\mp \prime} z_{i}^{ \pm} z_{i}^{ \pm \prime}\right\rangle+v\left\langle z_{k}^{\mp \prime} \partial_{m}^{\prime} \partial_{m}^{\prime}\left(z_{i}^{ \pm} z_{i}^{ \pm \prime}\right)\right\rangle+v\left\langle z_{i}^{ \pm} z_{i}^{ \pm \prime} \partial_{m}^{\prime} \partial_{m}^{\prime} z_{k}^{\mp \prime}\right\rangle
\end{aligned}
$$

It is evident that $\boldsymbol{B}_{0}$ appears explicitly here, in contrast to (3.11). Therefore, the entire influence of the mean magnetic field on the energy distribution, and upon spectral transfer, must proceed through its influence on the solutions of (5.1). This influence consists of the explicit effects of the terms involving $B_{0}$ as well as the implicit influence of $B_{0}$ on the fourth-order terms appearing in (5.1), as is apparent in the 
evolution equations for these higher-order correlations (not written here). In light of this, we now turn to a discussion of a few elementary ways in which correlations, including fourth-order correlations, can influence the varieties of MHD turbulence that emerge from the dynamics.

\section{Evidence of several varieties of MHD turbulence based on global properties of initial conditions}

Another feature of MHD that sets it apart from incompressible 3D hydrodynamic turbulence is the presence of more than one (relevant) ideal quadratic global invariant. Note that here we exclude another well-known invariant in 3D hydrodynamics, the kinetic helicity, as its role in energy decay is unclear (Kraichnan 1973). In homogeneous (or periodic) 3D MHD with no mean magnetic field, three quantities are conserved in the absence of viscosity and resistivity and forcing, namely the energy $E$, the cross-helicity $H_{c}$, and the magnetic helicity $H_{m}=\langle\boldsymbol{a} \cdot \boldsymbol{b}\rangle$, where $\boldsymbol{b}=\boldsymbol{\nabla} \times \boldsymbol{a}$. (See also Politano et al. 2003.) When dissipative effects are reinstated, each of these quantities is expected to have a conserved spectral flux in the inertial range. Measures of the relative amount of these bulk quantities are the dimensionless ratios $\sigma_{c}=\left(Z_{+}^{2}-Z_{-}^{2}\right) /\left(Z_{+}^{2}+Z_{-}^{2}\right)$ associated with $H_{c}$, and $\sigma_{m}=\left(Z_{L}^{2}-Z_{R}^{2}\right) /\left(Z_{L}^{2}+Z_{R}^{2}\right)$ associated with magnetic helicity. Here $Z_{L}^{2}$ and $Z_{R}^{2}$ are magnetic energies for left- and right-handed structures, respectively (Matthaeus 1999). In terms of the integrated magnetic helicity spectrum $H_{m}(\boldsymbol{k})$,

$$
\begin{aligned}
& Z_{L}^{2}=\frac{1}{2}\left[E_{b}+\int \mathrm{d}^{3} k|\boldsymbol{k}| H_{m}(\boldsymbol{k})\right], \\
& Z_{R}^{2}=\frac{1}{2}\left[E_{b}-\int \mathrm{d}^{3} k|\boldsymbol{k}| H_{m}(\boldsymbol{k})\right],
\end{aligned}
$$

where $E_{b}$ is the total magnetic energy.

It is already well known (Stribling \& Matthaeus 1991) from simulation studies and from associated relaxation theories in 3D MHD (see also Ting, Matthaeus \& Montgomery (1986) for the two-dimensional case) that MHD turbulence with differing values of $\sigma_{c}$ and $\sigma_{m}$ can experience very different relaxation processes during turbulence decay. Furthermore the energy itself is not of just one type in MHD, and the partitioning into kinetic energy $E^{v}$ and magnetic energy $E^{b}$ can cause dynamical behaviour as distinct as the kinematic dynamo, for $E^{v} \gg E^{b}$, and disruptivelike reconnection events for $E^{b} \gg E^{v}$. Therefore, the energy ratio $r_{A}=E^{v} / E^{b}$ appears as a distinguishing dimensionless parameter. Indeed the above-mentioned studies (Ting et al. 1986; Stribling \& Matthaeus 1991) have demonstrated that varying $\sigma_{m}, \sigma_{c}$ and $r_{A}$ in the initial data can lead to extremely different long-time behaviour. In some cases the selective decay process is favoured (Montgomery, Turner \& Vahala 1979; Matthaeus \& Montgomery 1980), and in some other cases dynamic alignment (Dobrowolny et al. 1980) is favoured. In still other cases MHD flow asymptotically reverts to a hydrodynamic flow dominated by kinetic energy. Given that the longterm properties of these MHD turbulence solutions differ so greatly, it is natural to suppose, based on the hypothesis of small-scale quasi-equilibrium (Batchelor 1970), that the cascades differ greatly in these different parameter regimes. Consequently one is obliged to adopt (at least) the set of parameters $\sigma_{c}, \sigma_{m}$ and $r_{A}$ as additional parameters that contribute to the labelling of different types of MHD turbulence.

A further important point concerning the diverse possibilities for turbulent relaxation in MHD can be made by examining the properties of the minimum energy states 
that are predicted for the final states of turbulent decay. These states include Alfvénic correlations $(\boldsymbol{v} \propto \boldsymbol{b})$, Beltrami correlations $(\boldsymbol{v} \propto \nabla \times \boldsymbol{v})$ and force-free correlations $(\boldsymbol{b} \propto \nabla \times \boldsymbol{b})$. The appearance of these states was first discussed for very long time global evolution (Ting et al. 1986; Stribling \& Matthaeus 1991). However, more recent studies have shown that these correlations also occur in random spatial patches at earlier times (Servidio, Matthaeus \& Dmitruk 2008). It was argued that the existence of spatial patches with random signed correlations requires non-Gaussian statistics. As an example, in an MHD flow with zero cross-helicity $\langle\boldsymbol{v} \cdot \boldsymbol{b}\rangle=0$, the presence of random signed patches with $\boldsymbol{v} \propto \pm \boldsymbol{b}$ requires that quantities such as $\left\langle(\boldsymbol{v} \cdot \boldsymbol{b})^{2}\right\rangle$ must depart from their values for uncorrelated Gaussian vector fields $\boldsymbol{v}$ and $\boldsymbol{b}$. The emergence of these non-Gaussian $\boldsymbol{v}, \boldsymbol{b}$ correlations seems to be very common in MHD turbulence (Matthaeus et al. 2008). Similarly spatial patches of force-free or Beltrami states are also found in MHD turbulence, and these imply different non-Gaussian correlations. Taken together, the rapid and widespread appearance of these correlations imply a dynamical tendency towards suppression of nonlinearity (see also Grappin et al. 1982, 1983; Matthaeus \& Montgomery 1984; Pouquet, Meneguzzi \& Frisch 1986; Kraichnan \& Panda 1988; Boldyrev 2006). That is, these correlations reduce the effectiveness of the principal nonlinearities of the incompressible MHD equations, namely the terms $\boldsymbol{v} \times \boldsymbol{b}, \boldsymbol{j} \times \boldsymbol{b}$ and $\boldsymbol{v} \times \boldsymbol{\omega}$.

Bearing in mind the idea that certain fourth-order correlations may suppress specific nonlinearities in the dynamical equations, it is not difficult to envision that a selective suppression of particular nonlinearities might modify the nature of the turbulence cascade. In this spirit we re-examine the recent simulation results of Lee et al. (2010). This study found that initial conditions with the same energy spectrum and essentially identical values of the global ideal invariants $E, H_{c}$ and $H_{m}$ can lead to very different inertial range spectra and global decay rates. We offer an explanation for this here, suggesting that there is a variation of the fourth-order correlations in their initial data that drives the systems to different regions of the phase space, resulting in different types of cascade and decay. That is, the fourth-order correlations induce different kinds of MHD turbulence, rather than one universal kind.

To see this we must delve into the initial data of Lee et al. (2010) in a little more detail. Three different initial conditions were used in their three decaying runs. The initial velocity field was chosen to be the same, but the initial magnetic fields used were different, and were labelled as $\boldsymbol{b}^{I}$ (insulating), $\boldsymbol{b}^{C}$ (conducting) and $\boldsymbol{b}^{A}$ (alternative), respectively. All three cases initially have zero cross-helicity and magnetic helicity, and $\left\langle(\boldsymbol{j} \cdot \boldsymbol{b})^{2}\right\rangle=0$. However, the three cases differ in their values for

$$
\frac{\left\langle(\boldsymbol{v} \cdot \boldsymbol{b})^{2}\right\rangle}{\left\langle\boldsymbol{v}^{2}\right\rangle\left\langle\boldsymbol{b}^{2}\right\rangle},
$$

with this quantity equal to 0 (for $\left.\boldsymbol{b}=\boldsymbol{b}^{I}\right), 1 / 6\left(\boldsymbol{b}^{C}\right)$ and $1 / 2\left(\boldsymbol{b}^{A}\right)$, respectively. One would expect then that for the 'alternative' case, the rate of nonlinear evolution of the magnetic field would be reduced due to suppression of the inductive electric field. In contrast, for the 'insulating' case, the magnetic evolution would proceed at full strength. The 'conducting' case has an intermediate status. The Lee et al. (2010) results show that the unsuppressed induction equation, with its $\boldsymbol{v} \times \boldsymbol{b}^{I}$ nonlinearity, gives rise to much more amplification of the magnetic field and much steeper spectra than the other two cases. Of course the greater magnetic energy implies weaker velocity fields, which means that the strength of the advective nonlinearity in the momentum equation is then reduced. The 'alternative' case, with the most suppressed 
inductive electric field, gives rise to near equipartition of kinetic and magnetic energies and a spectrum closest to the Kolmogorov $-5 / 3$ spectral index prediction. It is thus reasonable to predict that varying the initial conditions, which at first glance are very similar but actually differ substantially in terms of at least one fourth-order statistic (6.3), leads to substantial changes in the subsequent cascade properties. Note that there is also the question as to whether currently attainable Reynolds numbers in numerical simulations are sufficient to generate genuine inertial ranges, or so-called minimum state flows. See Beresnyak (2011) and Zhou \& Oughton (2011) for further discussion.

Based on this analysis of the Lee et al. (2010) study, and the association of suppression of nonlinearity with fourth-order correlations, we are motivated to introduce the following hypothesis: we suggest that the fourth-order correlations that are linked with suppression of specific nonlinearities, namely $\left\langle|\boldsymbol{v} \cdot \boldsymbol{b}|^{2}\right\rangle,\left\langle\left.\boldsymbol{j} \cdot \boldsymbol{b}\right|^{2}\right\rangle$ and $\left\langle|\boldsymbol{v} \cdot \boldsymbol{\omega}|^{2}\right\rangle$, are also of significance in controlling the type of cascade. Forcing functions might be likewise classified depending on how they drive the correlations. We propose that the nature of MHD solutions obtained from a set of initial conditions or forcing functions can be strongly influenced by selection of the strength of these correlations. Accordingly, it is convenient to consider the normalized correlations,

$$
\begin{aligned}
\Sigma_{v b} & \equiv \frac{\left\langle(\boldsymbol{v} \cdot \boldsymbol{b})^{2}\right\rangle}{\left\langle\boldsymbol{v}^{2}\right\rangle\left\langle\boldsymbol{b}^{2}\right\rangle}, \\
\Sigma_{j b} & \equiv \frac{\left\langle(\boldsymbol{j} \cdot \boldsymbol{b})^{2}\right\rangle}{\left\langle\boldsymbol{j}^{2}\right\rangle\left\langle\boldsymbol{b}^{2}\right\rangle}, \\
\Sigma_{v \omega} & \equiv \frac{\left\langle(\boldsymbol{v} \cdot \boldsymbol{\omega})^{2}\right\rangle}{\left\langle\boldsymbol{v}^{2}\right\rangle\left\langle\boldsymbol{\omega}^{2}\right\rangle},
\end{aligned}
$$

which provide a measure of the strength of each of these correlations.

The effectiveness of the control of the solutions exerted by $\Sigma_{v b}, \Sigma_{j b}$ and $\Sigma_{v \omega}$ remains to be established by further computations. However, one may readily see the pathway for their influence by inspecting the right-hand side of (5.1), which contains several fourth-order moments. Variation in the strength of $\Sigma_{v b}, \Sigma_{j b}$ and $\Sigma_{v \omega}$ will place constraints, through Schwartz inequalities, on these terms that drive the evolution of the third-order moments. The third-order moments, in turn, drive the evolution of the spectrum through (2.4). This is the chain of control of spectral transfer due to these 'suppressing' correlations, and also due to the mean magnetic field $\boldsymbol{B}_{0}$, various helicities, and any other dynamically important quantities that appear in the higher-order moment equations, but which are absent in the second-order von Kármán-Howarth equation and absent in the universal form of the third-order law (3.11).

\section{Likelihood of non-universality in decaying and steady driven MHD turbulence}

In isotropic hydrodynamic turbulence, there are two von Kármán-Howarth similarity variables: the turbulence energy $u^{2}$ and a similarity scale $L$. The dimensionless correlation functions then depend only on a dimensionless length scale $\eta=r / L$. This approach is valid for scales $r \gg \lambda_{\text {diss }}=\left(v^{3} / \epsilon\right)^{1 / 4}$, and can accommodate $\eta \sim 1$. However, for very high Reynolds numbers the dissipation scale becomes very small, and it is also possible that $1 \gg \eta \gg \lambda_{\text {diss }}$. There then arises the possibility of an inertial range of scales in which the properties are self-similar. In this sense the only parameter that appears is the Reynolds number, and when it takes on an asymptotically 
large value, a 'universal' theory can emerge that may apply over a wide range of (inertial) range values of $\eta$. The question at hand is whether such a regime of universal behaviour is likely to occur in MHD turbulence. In fact, it is apparent that if such a development is valid for MHD, there are likely to be at least several and possibly many such regimes. We develop this argument as follows.

First let us consider isotropic MHD turbulence. It is sensible to suppose that one should investigate asymptotic regimes of large Reynolds number $R e$ and magnetic Reynolds number $R m$, in analogy to the standard hydrodynamic case. However, the ratio of these, the magnetic Prandtl number $P r=R e / R m$ can take on any value, and so it remains a free parameter in the search for universality. Based on the discussion in the previous sections, the ratio of the two similarity lengths $L^{+} / L^{-}$is another dimensionless number, of arbitrary magnitude, that can enter the description. Another well-known feature of MHD is that the relative amount of kinetic energy $E^{v}$ and magnetic energy $E^{b}$ can vary greatly. For example when $E^{v} \gg E^{b}$ one might be in the kinematic dynamo regime (e.g. Schekochihin et al. 2002; Mininni, Alexakis \& Pouquet 2005; Tobias \& Cattaneo 2008), but when $E^{b} \gg E^{v}$ the dynamics may be dominated by Lorentz forces and magnetic reconnection. Therefore, one would expect that the Alfvén ratio $r_{A}=E^{v} / E^{b}$ would be an important factor in determining what kind of cascade behaviour is obtained. Also relevant are the normalized cross-helicity $\sigma_{c}$ and the normalized magnetic helicity $\sigma_{m}$. There is no analogue of these three ratios, $\sigma_{c}, \sigma_{m}$ and $r_{A}$ in the description the evolution of incompressible Navier-Stokes turbulence. Therefore, there is no reason to suspect that a single universal theory would be applicable when $R e \rightarrow \infty$ and $R m \rightarrow \infty$ without regard to the values taken by the two parameters $\operatorname{Pr}$ and $L^{+} / L^{-}$.

So as an exercise, let us estimate the possibilities by counting possible values of the ratios $\operatorname{Pr}, L^{+} / L^{-}$and $r_{A}$ as threefold: high values $(\gg 1)$, order unity and low values $(\ll 1)$. Similarly (and very roughly speaking) the second-order dimensionless parameters $\sigma_{c}$ and $\sigma_{m}$ can take on minimal values $(\approx 0)$, maximal values (near \pm 1 ), or intermediate values. The same argument can be made for the three fourth-order dimensionless parameters $\Sigma_{v b}, \Sigma_{j b}$ and $\Sigma_{v \omega}$. Allowing for the possibility that distinct combinations of these parameters may lead to distinct cascade properties, we are led to the conclusion that for isotropic incompressible 3D MHD turbulence there are at least $\sim 3^{8} \approx 6500$ conceptually distinct types of possible turbulent behaviour. Of course, it is possible that many types may have very similar actual behaviour, sometimes referred to as 'universality within classes' (e.g. Schekochihin, Cowley \& Yousef 2008).

We may also carry out this counting exercise for the case of MHD turbulence that is anisotropic due to the presence of a uniform DC magnetic field. The reasoning is similar to the case above, with several adjustments. There are now three dimensionless ratios of lengths $L_{\perp}^{-} / L_{\perp}^{+}, L_{\|}^{-} / L_{\perp}^{+}$and $L_{\|}^{+} / L_{\perp}^{+}$. (If the dynamics is described by a similarity law, then according to the reasoning presented above the last of these two may not be dynamically independent. However, the ratios of parallel to perpendicular length scales, even if constant, may still modify the cascade. Therefore, we count the three length scale ratios in all cases.) For this case we have an additional parameter, $\delta B / B_{0}$, the ratio of the r.m.s. magnetic fluctuations (the turbulence amplitude) $\delta B$ to the DC magnetic field strength $B_{0}$. The cross-helicity and $\sigma_{c}$ remain of relevance, but since magnetic helicity is not conserved in this case, $\sigma_{m}$ is no longer included in the accounting. Finally, once again we have the three dimensionless parameters associated with fourth-order correlations (6.4)-(6.6). This could give at least 10 parameters that control the cascade, leading to $\sim 3^{10} \approx 59000$ potentially distinct varieties of anisotropic MHD turbulent cascades, although again universality within a considerably 
smaller number of classes is possible, perhaps even likely. It would be very interesting to perform simulation studies to investigate how many of these potentially different turbulent cascades occur.

The extent to which MHD turbulence is universal is a topic currently enjoying debate (e.g. Schekochihin et al. 2008; Grappin \& Müller 2010; Lee et al. 2010; Beresnyak 2011; Boldyrev et al. 2011; Mininni 2011; Pouquet et al. 2011). Unfortunately, many of the simulations studies do not report values for the fourthorder correlations, making it difficult to discuss their results in connection with our hypothesis about the importance of such correlations. As noted in $\S 6$, the Lee et al. (2010) simulations are consistent with our hypothesis. In a future paper, we will report on a simulation study where fourth-order correlations are varied. For a recent review of the related topic of locality of interactions in MHD see Mininni (2011).

\section{Conclusions}

We have established, using analysis of the type introduced by de Kármán \& Howarth (1938), that a similarity decay of isotropic 3D MHD can be described by a single length scale when the normalized cross-helicity $\left(\sigma_{c}\right)$ is constant. However, when $\sigma_{c}$ is time-varying, we have only been able to find similarity decay with two independent length scales. When a uniform DC magnetic field $\left(B_{0}\right)$ is also present, we can expect that there will be four relevant energy-containing scales, a parallel and perpendicular one for each of $Z^{ \pm}$. In fact, the analysis for the prospects of a similarity decay in this case indicates that the two parallel length scales can be initially chosen independently, but similarity requires that they do not evolve independently of the perpendicular length scales, at least for the forms of similarity considered herein. Therefore, the parallel length scales remain as independent initial parameters, but they will not be independent similarity variables. For both the $B_{0}=0$ (with non-constant $\sigma_{c}$ ) case and the $B_{0} \neq 0$ case, self-similar decay with only one independent similarity length scale does not appear to be possible. In both cases, however, including two similarity length scales does allow for self-similar decay.

In addition we have pointed out that with a uniform DC magnetic field, the functional form of the second-order von Kármán-Howarth equation is unchanged. This means that the influence of the DC field on the dynamics of the spectrum is only through the implicit dependence of the third-order moments on the DC field. The DC field enters explicitly in the equation for the time evolution of the third-order moments, and thus affects the lifetime of the triple correlations, as has long been argued on physical grounds.

Turning to the fourth-order moments, we draw a connection between their structure and the phenomenon of rapid suppression of nonlinearity. This is caused by spontaneous development of spatial patches of correlations that reduce the strength of nonlinear terms in the MHD equations. Appearance of these patches requires that the statistical distributions be non-Gaussian. Furthermore, we suggest that initial values of certain fourth-order correlations (as well as driving forces that induce these correlations), can selectively control the strength of different nonlinearities. It is plausible that this fourth-order statistical effect is responsible for differences in cascade behaviour observed, for example, in the recent study of Lee et al. (2010).

Finally we discussed how the multiplicity of length scales, ideal invariants, different modes of fourth-order suppression, and other parameters can conceivably give rise to thousands of varieties of MHD turbulence. Under these circumstances it seems unlikely that efforts would succeed in finding a single 'universal' law for MHD turbulence cascade, although the possibility of universality within a relatively small 
number of classes remains open. It is interesting to speculate that there may also be many varieties of Navier-Stokes turbulence, due, for example, to the influence of rotation, kinetic helicity, $\Sigma_{v \omega}$ and other higher-order correlations.

\section{Acknowledgements}

This research is supported in part by NSF Solar Terrestrial Program Grant AGS1063439, NSF SHINE grant ATM-0752135, NASA Heliophysics grants NNX09AH79, NNX09AG31G and NNX11AJ44G and the Strategic Research Investment Fund at the University of Waikato. We acknowledge the Marie Curie Project FP7 PIRSES-2010269297 - "Turboplasmas."

\section{Appendix. Evolution equations for structure functions}

Subtracting (2.1) from (2.2) gives the following equation for the Elsässer increments, $\delta z^{ \pm}=z^{ \pm}\left(x^{\prime}\right)-z^{ \pm}(x)$

$$
\partial_{t} \delta z_{i}^{ \pm}=-\delta z_{k}^{\mp} \partial_{k}^{\prime} \delta z_{i}^{ \pm}-\left(z_{k}^{\mp} \mp B_{0 k}\right)\left(\partial_{k}^{\prime}+\partial_{k}\right) \delta z_{i}^{ \pm}-\left(\partial_{i}^{\prime}+\partial_{i}\right) \delta P+v\left(\partial_{k}^{\prime} \partial_{k}^{\prime}+\partial_{k} \partial_{k}\right) \delta z_{i}^{ \pm},
$$

where we use the property that the primed and unprimed coordinates are independent, so that $\partial_{k} z_{i}^{ \pm \prime}=0$ and $\partial_{k}^{\prime} z_{i}^{ \pm}=0$.

Multiplying this by $2 \delta z_{i}^{ \pm}$and averaging yields

$$
\partial_{t}\left\langle\left|\delta z_{i}^{ \pm}\right|^{2}\right\rangle=-\frac{\partial}{\partial r_{k}}\left\langle\delta z_{k}^{\mp}\left|\delta z_{i}^{ \pm}\right|^{2}\right\rangle+2 v \frac{\partial^{2}}{\partial r_{k}^{2}}\left\langle\left|\delta z_{i}^{ \pm}\right|^{2}\right\rangle-4 v\left\langle\left|\partial_{k} z_{i}^{ \pm}\right|^{2}\right\rangle .
$$

In arriving at this expression we make use of $\partial_{k}\langle\bullet\rangle=-\left(\partial / \partial r_{k}\right)\langle\bullet\rangle$ and $\partial_{k}^{\prime}\langle\bullet\rangle=$ $\left(\partial / \partial r_{k}\right)\langle\bullet\rangle$. These latter relations follow from spatial homogeneity. Note the absence of any (explicit) dependence on $\boldsymbol{B}_{0}$.

For a steady-state, high-Reynolds-number flow, this equation is the famous thirdorder law:

$$
\frac{\partial}{\partial r_{k}}\left\langle\delta z_{k}^{\mp}\left|\delta z_{i}^{ \pm}\right|^{2}\right\rangle=-4 \epsilon^{ \pm},
$$

where $\epsilon^{ \pm}=v\left\langle\left|\partial_{k} z_{i}^{ \pm}\right|^{2}\right\rangle$. If the flow is isotropic, as might occur in the absence of a DC magnetic field $\boldsymbol{B}_{0}$, then (A 3 ) reduces to the more familiar form

$$
\left\langle\delta z_{L}^{\mp}\left|\delta z_{i}^{ \pm}\right|^{2}\right\rangle=-\frac{4}{3} \epsilon^{ \pm} r,
$$

where $\delta z_{L}^{ \pm}=\hat{\boldsymbol{r}} \cdot \delta z^{ \pm}$are the longitudinal Elsässer increments.

Similarly, we can derive an equation for the evolution of the third-order structure function $\left\langle\delta z_{k}^{\mp}\left|\delta z_{i}^{ \pm}\right|^{2}\right\rangle$ :

$$
\begin{aligned}
\partial_{t}\left\langle\delta z_{k}^{\mp}\left|\delta z_{i}^{ \pm}\right|^{2}\right\rangle= & -\frac{\partial}{\partial r_{m}}\left\langle\delta z_{m}^{\mp} \delta z_{k}^{\mp}\left|\delta z_{i}^{ \pm}\right|^{2}\right\rangle+\left\langle\left|\delta z_{i}^{ \pm}\right|^{2}\left(\delta z_{m}^{\mp}-\delta z_{m}^{ \pm}\right) \partial_{m}^{\prime} \delta z_{k}^{\mp}\right\rangle \\
& +\left\langle\left|\delta z_{i}^{ \pm}\right|^{2}\left(z_{m}^{\mp}-z_{m}^{ \pm}\right) \partial_{m}^{\prime} \delta z_{k}^{\mp}\right\rangle \mp 2 B_{0 m}\left\langle\left|\delta z_{i}^{ \pm}\right|^{2}\left(\partial_{m}^{\prime}+\partial_{m}\right) \delta z_{k}^{\mp}\right\rangle \\
& -2\left\langle\delta z_{k}^{\mp} \delta z_{i}^{ \pm}\left(\partial_{i}^{\prime}+\partial_{i}\right) \delta P\right\rangle-\left\langle\left|\delta z_{i}^{ \pm}\right|^{2}\left(\partial_{k}^{\prime}+\partial_{k}\right) \delta P\right\rangle \\
& +2 v\left\langle\delta z_{k}^{\mp} \delta z_{i}^{ \pm}\left(\partial_{m}^{\prime} \partial_{m}^{\prime}+\partial_{m} \partial_{m}\right) \delta z_{i}^{ \pm}\right\rangle \\
& +2 v\left\langle\left|\delta z_{i}^{ \pm}\right|^{2}\left(\partial_{m}^{\prime} \partial_{m}^{\prime}+\partial_{m} \partial_{m}\right) \delta z_{k}^{\mp}\right\rangle .
\end{aligned}
$$

Clearly, this depends explicitly (and implicitly) on $\boldsymbol{B}_{0}$. 


\section{REFERENCES}

AleXAKIs, A. 2007 Nonlocal phenomenology for anisotropic magnetohydrodynamic turbulence. Astrophys. J. 667, L93-L96.

Aluie, H. \& Eyink, G. L. 2010 Scale locality of magnetohydrodynamic turbulence. Phys. Rev. Lett. 104, 081101.

Barenblatt, G. I. 1996 Scaling, Self-similarity, and Intermediate Asymptotics. Cambridge University Press.

Batchelor, G. K. 1970 The Theory of Homogeneous Turbulence. Cambridge University Press.

Beresnyak, A. 2011 Spectral slope and Kolmogorov constant of MHD turbulence. Phys. Rev. Lett. 106, 075001.

Beresnyak, A. \& LAzArian, A. 2008 Strong imbalanced turbulence. Astrophys. J. 682, 1070-1075.

Beresnyak, A. \& LAZARIAn, A. 2010 Scaling laws and diffuse locality of balanced and imbalanced magnetohydrodynamic turbulence. Astrophys. J. 722, L110-L113.

Biskamp, D. 1994 Cascade models for magnetohydrodynamic turbulence. Phys. Rev. E 50, 2702.

Boldyrev, S. 2006 Spectrum of magnetohydrodynamic turbulence. Phys. Rev. Lett. 96, 115002.

Boldyrev, S., Perez, J. C., Borovsky, J. E. \& Podesta, J. J. 2011 Spectral scaling laws in magnetohydrodynamic turbulence simulations and in the solar wind. Astrophys. J. 741, L19.

Bondeson, A. 1985 Cascade properties of shear Alfvén turbulence. Phys. Fluids 28, 2406-2411.

CArbone, V., Sorriso-Valvo, L. \& Marino, R. 2009 On the turbulent energy cascade in anisotropic magnetohydrodynamic turbulence. Europhys. Lett. 88, 25001.

CARbone, V. \& Veltri, P. 1990 A shell model for anisotropic magnetohydrodynamic turbulence. Geophys. Astrophys. Fluid Dyn. 52, 153-181.

Chandran, B. D. G. 2008 Strong anisotropic MHD turbulence with cross helicity. Astrophys. J. 685, 646-658.

CHANDRASEKHAR, S. 1951 $a$ The invariant theory of isotropic turbulence in magneto-hydrodynamics. Proc. R. Soc. Lond. A 204, 435.

ChANDRASEKHAR, S. $1951 b$ The invariant theory of isotropic turbulence in magneto-hydrodynamics. II. Proc. R. Soc. Lond. A 207, 301.

Cho, J. \& VishniAc, E. T. 2000 The anisotropy of magnetohydrodynamic Alfvénic turbulence. Astrophys. J. 539, 273-282.

DAVIDSON, P. A. 2009 The role of angular momentum conservation in homogeneous turbulence. J. Fluid Mech. 632, 329-358.

DAVIDSON, P. A. 2010 On the decay of Saffman turbulence subject to rotation, stratification or an imposed magnetic field. J. Fluid Mech. 663, 268-292.

Dobrowolny, M., Mangeney, A. \& Veltri, P. 1980 Fully developed anisotropic hydromagnetic turbulence in interplanetary space. Phys. Rev. Lett. 45, 144-147.

Domaradzki, J. A., TeaCA, B. \& CARATI, D. 2010 Locality properties of the energy flux in magnetohydrodynamic turbulence. Phys. Fluids 22, 051702.

Dryden, H. L. 1943 A review of the statistical theory of turbulence. Q. Appl. Maths 1, 7.

FRISCH, U. 1995 Turbulence. Cambridge University Press.

GAltiER, S. 2008 von Kármán-Howarth equations for Hall magnetohydrodynamic flows. Phys. Rev. E 77, 015302.

Galtier, S., Politano, H. \& Pouquet, A. 1997 Self-similar energy decay in magnetohydrodynamic turbulence. Phys. Rev. Lett. 79, 2807.

Galtier, S., Zienicke, E., Politano, H. \& Pouquet, A. 1999 Parametric investigation of self-similar decay laws in MHD turbulent flows. J. Plasma Phys. 61, 507.

Goldreich, P. \& SRIDhAR, S. 1995 Toward a theory of interstellar turbulence: II. Strong Alfvénic turbulence. Astrophys. J. 438, 763-775.

GRAPPIN, R. 1986 Onset and decay of two-dimensional magnetohydrodynamic turbulence with velocity-magnetic field correlation. Phys. Fluids 29, 2433-2443.

Grappin, R., Frisch, U., LÉORAT, J. \& Pouquet, A. 1982 Alfvénic fluctuations as asymptotic states of MHD turbulence. Astron. Astrophys. 105, 6-14. 
GrapPIN, R. \& MÜLlER, W.-C. 2010 Scaling and anisotropy in magnetohydrodynamic turbulence in a strong mean magnetic field. Phys. Rev. E 82 (2), 026406.

Grappin, R., Pouquet, A. \& LÉorat, J. 1983 Dependence of MHD turbulence spectra on the velocity field-magnetic field correlation. Astron. Astrophys. 126, 51-58.

Hossain, M., Gray, P. C., Pontius, D. H. JR., Matthaeus, W. H. \& Oughton, S. 1995 Phenomenology for the decay of energy-containing eddies in homogeneous MHD turbulence. Phys. Fluids 7, 2886-2904.

IROSHNiKOV, R. S. 1963 Turbulence of a conducting fluid in a strong magnetic field. Astron. Zh. 40, 742 (Sov. Astron.7, 566-571 (1964)).

de Kármán, T. \& Howarth, L. 1938 On the statistical theory of isotropic turbulence. Proc. $R$. Soc. Lond. Ser. A 164, 192-215.

VON KÁRMÁn, T. \& LIN, C. C. 1949 On the concept of similarity in the theory of isotropic turbulence. Rev. Mod. Phys. 21, 516.

Kolmogorov, A. N. 1941a Local structure of turbulence in an incompressible viscous fluid at very high Reynolds numbers. Dokl. Akad. Nauk SSSR 30, 301-305 (Reprinted in Proc. R. Soc. Lond. Ser. A 434, 9-13 (1991)).

Kolmogorov, A. N. $1941 b$ On degeneration of isotropic turbulence in an incompressible viscous liquid. C.R. Acad. Sci. 31, 538-540.

Kolmogorov, A. N. 1941c Dissipation of energy in the locally isotropic turbulence. C.R. Acad. Sci. 32, 16 (Reprinted in Proc. R. Soc. Lond. Ser. A 434, 15-17 (1991)).

Kraichnan, R. H. 1965 Inertial-range spectrum of hydromagnetic turbulence. Phys. Fluids 8, $1385-1387$.

Kraichnan, R. H. 1973 Helical turbulence and absolute equilibrium. J. Fluid Mech. 59, 745.

KRAichnan, R. H. \& PANDA, R. 1988 Depression of nonlinearity in decaying isotropic turbulence. Phys. Fluids 31, 2395-2397.

Lee, E., Brachet, M. E., Pouquet, A., Mininni, P. D. \& Rosenberg, D. 2010 Lack of universality in decaying magnetohydrodynamic turbulence. Phys. Rev. E 81, 016318.

Lithwick, Y., Goldreich, P. \& SRIdhar, S. 2007 Imbalanced strong MHD turbulence. Astrophys. J. 655, 269-274.

MacBride, B. T., Forman, M. A. \& SMith, C. W. 2005 Turbulence and third moment of fluctuations: Kolmogorov's 4/5 law and its MHD analogues in the solar wind. In Proceedings Solar Wind 11 - Soho 16 Connecting Sun and Heliosphere, vol. SP-592 (ed. B. Fleck, T.H. Zurbuchen \& H. Lacoste). pp. 613-616. European Space Agency.

Matthaeus, W. H. 1999 Magnetic helicity and homogeneous turbulence models. In Geophysical Monograph 111. Proceedings of Magnetic Helicity in Space and Laboratory Plasmas (ed. M. R. Brown, R. C. Canfield \& A. A. Pevtsov). pp. 247-255. AGU.

Matthaeus, W. H. \& Montgomery, D. 1980 Selective decay hypothesis at high mechanical and magnetic Reynolds numbers. Ann. N. Y. Acad. Sci. 357, 203-222.

Matthaeus, W. H. \& Montgomery, D. 1984 Dynamic alignment and selective decay in MHD. In Statistical Physics and Chaos in Fusion Plasmas (ed. C. W. Jr. Horton \& L. E. Reichl), pp. 285-291. Wiley.

Matthaeus, W. H., Oughton, S., Pontius, D. \& Zhou, Y. 1994 Evolution of energy containing turbulent eddies in the solar wind. J. Geophys. Res. 99, 19267-19287.

Matthaeus, W. H., Pouquet, A., Mininni, P. D., Dmitruk, P. \& Breech, B. 2008 Rapid alignment of velocity and magnetic field in magnetohydrodynamic turbulence. Phys. Rev. Lett. 100, 085003.

Matthaeus, W. H. \& SMith, C. 1981 Structure of correlation tensors in homogeneous anisotropic turbulence. Phys. Rev. A 24, 2135-2144.

Matthaeus, W. H. \& ZhOU, Y. 1989 Extended inertial range phenomenology of magnetohydrodynamic turbulence. Phys. Fluids B 1, 1929-1931.

Milano, L. J., Matthaeus, W. H., Dmitruk, P. \& Montgomery, D. C. 2001 Local anisotropy in incompressible magnetohydrodynamic turbulence. Phys. Plasmas 8, 2673-2681.

Mininni, P. D. 2011 Scale interactions in magnetohydrodynamic turbulence. Ann. Rev. Fluid Mech. 43 (1), 377-397. 
Mininni, P., Alexakis, A. \& Pouquet, A. 2005 Shell-to-shell energy transfer in magnetohydrodynamics. II. Kinematic dynamo. Phys. Rev. E 72, 046302.

Montgomery, D. C. 1982 Major disruption, inverse cascades, and the Strauss equations. Phys. Scr. T2/1, 83-88.

Montgomery, D., Turner, L. \& VAhala, G. 1979 Most probable states in magnetohydrodynamics. J. Plasma Phys. 21, 239.

Oвukhov, A. M. 1941 On the energy distribution in the spectrum of a turbulent flow. Dokl. Akad. Nauk SSSR 32, 22-24 (C.R. (Dokl.) Acad. Sci. URSS 32, 19 (1963)).

Oughton, S., Priest, E. R. \& Matthaeus, W. H. 1994 The influence of a mean magnetic field on three-dimensional MHD turbulence. J. Fluid Mech. 280, 95-117.

Perez, J. C. \& BoldyreV, S. 2009 Role of cross-helicity in magnetohydrodynamic turbulence. Phys. Rev. Lett. 102, 025003.

PodestA, J. J. 2008 Laws for third-order moments in homogeneous anisotropic incompressible magnetohydrodynamic turbulence. J. Fluid Mech. 609, 171-194.

Podesta, J. J., Forman, M. A. \& Smith, C. W. 2007 Anisotropic form of third-order moments and relationship to the cascade rate in axisymmetric magnetohydrodynamic turbulence. Phys. Plasmas 14 (9), 092305.

Politano, H., Gomez, T. \& Pouquet, A. 2003 von Kármán-Howarth relationship for helical magnetohydrodynamic flows. Phys. Rev. E 68 (2), 026315.

Politano, H. \& Pouquet, A. $1998 a$ Dynamical length scales for turbulent magnetized flows. Geophys. Res. Lett. 25, 273-276.

Politano, H. \& Pouquet, A. $1998 b$ von Kármán-Howarth equation for magnetohydrodynamics and its consequences on third-order longitudinal structure and correlation functions. Phys. Rev. E 57, R21.

Politano, H., Pouquet, A. \& Sulem, P. L. 1995 Current and vorticity dynamics in three-dimensional magnetohydrodynamic turbulence. Phys. Plasmas 2, 2931.

Pouquet, A., Brachet, M.-E., Lee, E., Mininni, P., Rosenberg, D. \& Uritsky, V. 2011 Lack of universality in MHD turbulence, and the possible emergence of a new paradigm? In Astrophysical Dynamics: From Stars to Galaxies, Proceedings IAU Symposium S271, vol. 6. pp. 304-316. Cambridge University Press.

Pouquet, A., Frisch, U. \& LÉorat, J. 1976 Strong MHD helical turbulence and the nonlinear dynamo effect. J. Fluid Mech. 77, 321-354.

Pouquet, A., Meneguzzi, M. \& Frisch, U. 1986 Growth of correlations in magnetohydrodynamic turbulence. Phys. Rev. A 33, 4266-4275.

Robertson, H. P. 1940 The invariant theory of isotropic turbulence. Proc. Camb. Phil. Soc. 36, 209.

Robinson, D. C. \& Rusbridge, M. G. 1971 Structure of turbulence in the zeta plasma. Phys. Fluids 14, 2499-2511.

Schekochihin, A. A., Cowley, S. C., Hammett, G. W., Maron, J. L. \& McWilliams, J. C. 2002 A model of nonlinear evolution and saturation of the turbulent MHD dynamo. New J. Phys. 4, 84.1-84.22.

Schekochihin, A. A., Cowley, S. C. \& Yousef, T. A. 2008 MHD turbulence: nonlocal, anisotropic, nonuniversal? In Computational Physics and New Perspectives in Turbulence (ed. Y. Kaneda), vol. 4. pp. 347-354. Springer.

Servidio, S., Matthaeus, W. H. \& Dmitruk, P. 2008 Depression of nonlinearity in decaying isotropic MHD turbulence. Phys. Rev. Lett. 100, 095005.

Shebalin, J. V., Matthaeus, W. H. \& Montgomery, D. 1983 Anisotropy in MHD turbulence due to a mean magnetic field. J. Plasma Phys. 29, 525-547.

Smith, C. W. 1981 The structure of axisymmetric turbulence. PhD thesis, College of William and Mary, Williamsburg, VA 23185.

Sorriso-Valvo, L., Marino, R., Carbone, V., Noullez, A., Lepreti, F., Veltri, P., Bruno, R., Bavassano, B. \& Pietropaolo, E. 2007 Observation of inertial energy cascade in interplanetary space plasma. Phys. Rev. Lett. 99, 115001.

Speziale, C. G. \& BERnARD, P. S. 1992 The energy decay in self-preserving isotropic turbulence revisited. J. Fluid Mech. 241, 645-667. 
Stribling, T. \& Matthaeus, W. H. 1991 Relaxation processes in a low order three-dimensional magnetohydrodynamics model. Phys. Fluids B 3, 1848.

Tennekes, H. \& Lumley, J. L. 1972 A First Course in Turbulence. MIT.

Ting, A. C., Matthaeus, W. H. \& Montgomery, D. 1986 Turbulent relaxation processes in magnetohydrodynamics. Phys. Fluids 29, 3261.

Tobias, S. M. \& Cattaneo, F. 2008 Limited role of spectra in dynamo theory: Coherent versus random dynamos. Phys. Rev. Lett. 101, 125003.

Wan, M., Servidio, S., Oughton, S. \& Matthaeus, W. H. 2009 The third-order law for increments in magnetohydrodynamic turbulence with constant shear. Phys. Plasmas 16.

Zank, G. P. \& MAtThaeus, W. H. 1992 The equations of reduced magnetohydrodynamics. J. Plasma Phys. 48, 85-100.

Zhou, Y. \& OUghton, S. 2011 Nonlocality and the critical Reynolds numbers of the minimum state magnetohydrodynamic turbulence. Phys. Plasmas 18, 072304. 